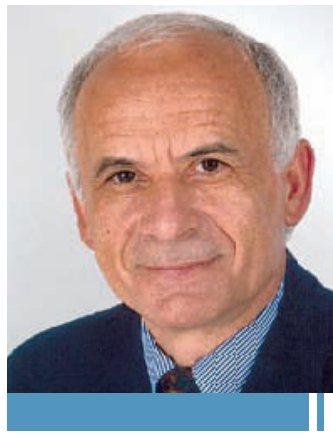

Prof. Dr. med. Alfred Wirth

Bad Rothenfelde

Beirat der Deutschen

Adipositas Gesellschaft

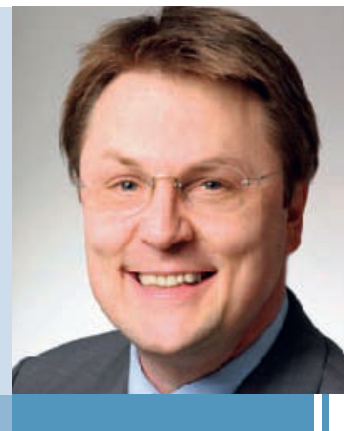

Prof. Dr. med. Jochen Seißler Diabetes-Zentrum, Medizinische Klinik Innenstadt, Klinikum der Universität München

Fettleber, Hypoglykämien \& Co

\title{
Behandlung häufiger Probleme bei Typ-2-Diabetikern
}

In der vorliegenden Ausgabe von Cardiovasc wollen wir mit Ihnen einige wichtige diagnostische und therapeutische Aspekte bei Patienten mit metabolischem Syndrom und Typ-2-Diabetes diskutieren. Angesichts der weltweiten Zunahme der Adipositas und des Typ-2Diabetes wird dringend nach neuen Therapieansätzen gesucht, die nachhaltig das Krankheitsbild bessern oder sogar heilen können.

Der erste Teil der Ausgabe beschäftigt sich mit der interessanten Fragestellung, welchen Stellenwert die bariatrische Chirurgie in der Therapie des Typ-2-Diabetes besitzt. Auch bei optimalen Therapievoraussetzungen gelingt weniger als 5\% der Betroffenen eine effektive langfristige Gewichtsabnahme. Von T. Horbach und A. Wirth werden in einer Pro und KontraDiskussion die Vorteile unterschiedlicher Verfahren aus der Sicht des Chirurgen und des Internisten dargestellt (S. 34, 37). Die metabolische Chirurgie zeigt bei den meisten Patienten, die mit konservativen Maßnahmen erfolglos "Typ-2-Diabetiker brauchen eine individuelle Therapie, die Alter, Diabetesdauer und Risikokonstellation berücksichtigt." behandelt worden sind, beeindruckende Erfolge hinsichtlich der Gewichtsreduktion und der Besserung der Stoffwechseleinstellung. Vergessen darf man aber auch nicht die Komplexität der einzelnen Eingriffe mit noch nicht genau absehbaren Spätfolgen und die notwendige engmaschige Nachsorge, die eine enge interdisziplinäre Kooperation notwendig macht. Die Schlussfolgerung der Autoren, dass die metabolische Chirurgie ausschließlich in ausgewiesenen erfahrenen Zentren erfolgen sollte, ist ein sehr wichtiger Punkt in der Beratung der Patienten.

Im zweiten Teil der vorliegenden Ausgabe werden spezielle Problemstellungen bei Menschen mit Typ-2-Diabetes dargestellt, die jedem niedergelassenen Kollegen in der Praxis häufig begegnen.

Bei Patienten mit Adipositas oder Diabetes mellitus wird sehr häufig in der Routineoberbauchsonografie die
Diagnose einer Fettleber gestellt. Der Beitrag von N. Stefan (S. 40) zeigt deutlich auf, dass die nichtalkoholische Fettleber ein unabhängiger Marker für hohes Atheroskleroserisiko und kardiovaskuläre Komplikationen darstellt. Bei Patienten mit Nachweis einer Fettleber ist deshalb in besonderem Maße geboten, eine regelmäßige vaskuläre Diagnostik und zielwertorientierte Therapie aller kardiovaskulären Risikofaktoren (Blutdruck, Lipide, Blutzucker) durchzuführen. Eine Gewichtsabnahme beseitigt die Fettleber und das metabolische Risiko.

Eine stärkere Beachtung der Hypoglykämien in der Behandlung von Typ-2-Diabetikern wird im Beitrag von T. Siegmund gefordert (S. 43). Herr Siegmund stellt neue Erkenntnisse zur Frage vor, wie intensiv Patienten mit Typ-2-Diabetes eingestellt werden sollten und welche bisher weitgehend unterschätzten Gefahren mit dem Auftreten von schweren Hypoglykämien verbunden sein können. Das Plädoyer für eine individuell gestaltete Therapie unter Berücksichtigung des Alters, der Diabetesdauer und der Risikokonstellation kann nur nachhaltig unterstützt werden. Im letzten Artikel wird von A. Lechner (S. 49) über den Umgang mit Patienten berichtet, bei denen eine Umstellung auf eine Insulintherapie notwendig ist. Die praktischen Tipps sollen helfen, die bewussten und unbewussten Ängste vor der Insulinspritze zu reduzieren, um eine gute Stoffwechseleinstellung beigleichzeitiger Therapiezufriedenheit zu fördern.

Wir hoffen, dass die Beiträge Ihnen einige hilfreiche Anregungen für die Behandlung Ihrer Patienten liefern und wünschen Ihnen viel Freude beim Lesen der Übersichtsartikel.

Ihre

Jochen Seißler und Alfred Wirth 